\title{
Editorial
}

\section{Advances in Statistical Channel Modeling for Wireless Communications}

\author{
José F. Paris, ${ }^{1}$ Paschalis C. Sofotasios, ${ }^{2,3}$ and Theodoros A. Tsiftsis ${ }^{4}$ \\ ${ }^{1}$ Department of Communications Engineering, University of Málaga, 29071 Málaga, Spain \\ ${ }^{2}$ Department of Electronics and Communications Engineering, Tampere University of Technology, 33720 Tampere, Finland \\ ${ }^{3}$ Department of Electrical and Computer Engineering, Aristotle University of Thessaloniki, 54124 Thessaloniki, Greece \\ ${ }^{4}$ Department of Electrical Engineering, Technological Educational Institute of Central Greece, 35100 Lamia, Greece
}

Correspondence should be addressed to José F. Paris; paris@ic.uma.es

Received 30 November 2014; Accepted 30 November 2014

Copyright (c) 2015 José F. Paris et al. This is an open access article distributed under the Creative Commons Attribution License, which permits unrestricted use, distribution, and reproduction in any medium, provided the original work is properly cited.

The complex electromagnetic propagation phenomena involved in modern wireless communications are taken into account by appropriate channel modeling. Statistical channel models are a powerful tool for communication engineers since they are able to capture the fundamental behavior of the wireless channel with reasonably simple mathematical formulation. New communication scenarios and services demand novel statistical channel models or extensions of those used in the field of wireless communications.

In the paper by J. Reig et al., the bivariate NakagamiLognormal model has been investigated. In particular, they have derived the joint probability density function, the cross moments, and the correlation coefficient in power terms. Also, two procedures to generate two correlated Nakagamilognormal random variables are described.

In the paper by J. Chen et al., a measurement campaign is introduced for modeling radio channels with either line-ofsight (LoS) or non-line-of-sight (NLoS) connection between user equipment (UE) and Node $\mathrm{B}(\mathrm{NB})$ in an operating universal mobile telecommunications system. A space alternating generalized expectation-maximization (SAGE) algorithm is applied to estimate the delays and the complex attenuations of multipath components from the obtained channel impulse responses.

In another paper by J. Reig et al., fading channels in the $60 \mathrm{GHz}$ band have been investigated. An exhaustive analysis of the small-scale fading amplitude in the $60 \mathrm{GHz}$ band is addressed for line-of-sight conditions (LOS). From a measurement campaign carried out in a laboratory, the authors have estimated the distribution of the small-scale fading amplitude over a bandwidth of $9 \mathrm{GHz}$.

In the paper by $\mathrm{C}$. Garcia-Corrales et al., the ergodic capacity of $\kappa-\mu$ shadowed fading channels is investigated. Considering the importance of the $\kappa-\mu$ shadowed distribution (it includes as special cases other classical distribution models such as one-side Gaussian, Rayleigh, Rician, etc.) the physical meaning of the distribution parameter variations and also their impact on the channel capacity is investigated. These results can be used to study the behavior of various channel models like the ones in underwater acoustic communications, land mobile satellite systems, body centric communications, and other wireless communication applications.

The paper by J. López-Fernández et al. addresses underwater electromagnetic communications, which are particularly important, although largely unexplored. Given that channel characterization and modelling in underwater communications differ substantially due to the different behavior of waves during subsea propagation, a suitable statistical model is proposed. The accuracy of this model is validated by comparisons with frequency response measurements and is shown to provide adequate fittings. Capitalizing on these results, the corresponding ergodic capacity is determined for a $7 \mathrm{MHz}$ bandwidth channel at distances ranging from $0.5 \mathrm{~m}$ 
to $5 \mathrm{~m}$ with different transmits power. This analysis shows capacity values of tens of kbps at distances of around $5 \mathrm{~m}$ to several Mbps at distances shorter than $1.5 \mathrm{~m}$.

In the paper by H. Jung and M. A. Weitnauer, cooperative transmissions (CT) formed by spatially separated wireless nodes which collaborate to form a virtual antenna array or virtual multiple-input-multiple-output (VMISO) link are studied. Unlike previous studies where the path loss of the VMISO link is studied as though the elements in the virtual antenna array are colocated, even though they are spread out, in this work the authors show that the spreading causes a signal-to-noise-ratio (SNR) penalty of up to $3 \mathrm{~dB}$. The analysis is also expanded for the high-SNR regime which showed a performance degradation caused by the path-loss disparity. Moreover, this degradation can be characterized equivalently by a log-normal distribution. Considering the above, a new CT link model expressed in closed form as an outage probability based on the log-normal shadowing model is proposed. The quality of the model is evaluated by a statistical test based on Kolmogorov-Smirnov method.

Given the extensive research activity in digital communication over fading channels over the past years, a thorough review by J. F. Paris presents the most important fading models that were proposed during the past decade. These models correspond to multipath, shadowing, and composite fading distributions and have been used extensively over the past years. The most important statistical measures of these models are also presented in a unified manner.

In the paper by W. Hao et al., energy-efficient resource allocation is investigated for a relay-based multiuser cooperation orthogonal frequency division multiple access (OFDMA) uplink system with amplify-and-forward (AF) protocol for all relays. An energy-efficient resource allocation problem that seeks joint optimization of subcarrier pairing, relay selection, subcarrier assignment, and power allocation is formulated. Unlike previous optimization throughput models, the considered EE problem is transformed into an equivalent optimal problem in subtractive form, which is solved by using dual decomposition and subgradient methods. More, two low-complexity suboptimal schemes to reduce computation costs are proposed. The simulation results show EE improvement of the energy-efficient design compared to that of the spectral-efficient design and the proposed suboptimal algorithm ordered subcarrier pairing (OSP) is close to the optimal algorithm.

In the paper by J. P. Peña-Martín and J. M. Romero-Jerez, novel closed-form expressions are derived for the bit error rate (BER) and channel capacity of a multiple-input-multipleoutput (MIMO) system in Rayleigh fading using transmit antenna selection (TAS) at the transmitter and maximal ratio combining (MRC) at the receiver.

Practical 2D and 3D wireless channel models multipolarized are proposed in the paper by K. Jeon et al. These models account for both cross polarization discrimination (XPD) and the Rician factor and are claimed to be simpler than existing models for MIMO systems. The offered results are subsequently employed in analyzing the corresponding error rate and achievable throughput for the case of equal-gain combining and maximum-ratio combining in multipolarized antenna systems.

José F. Paris

Paschalis C. Sofotasios

Theodoros A. Tsiftsis 

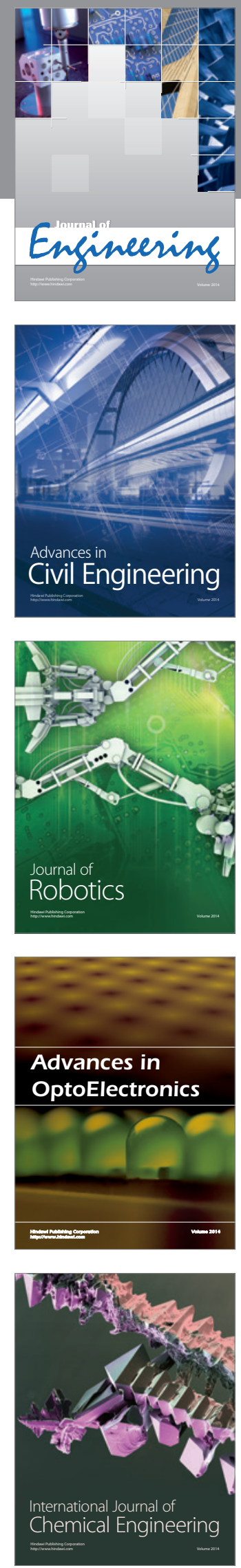

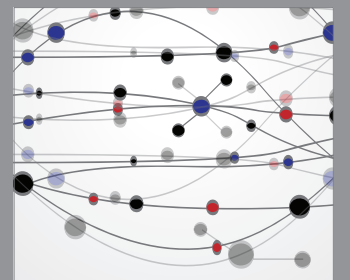

The Scientific World Journal
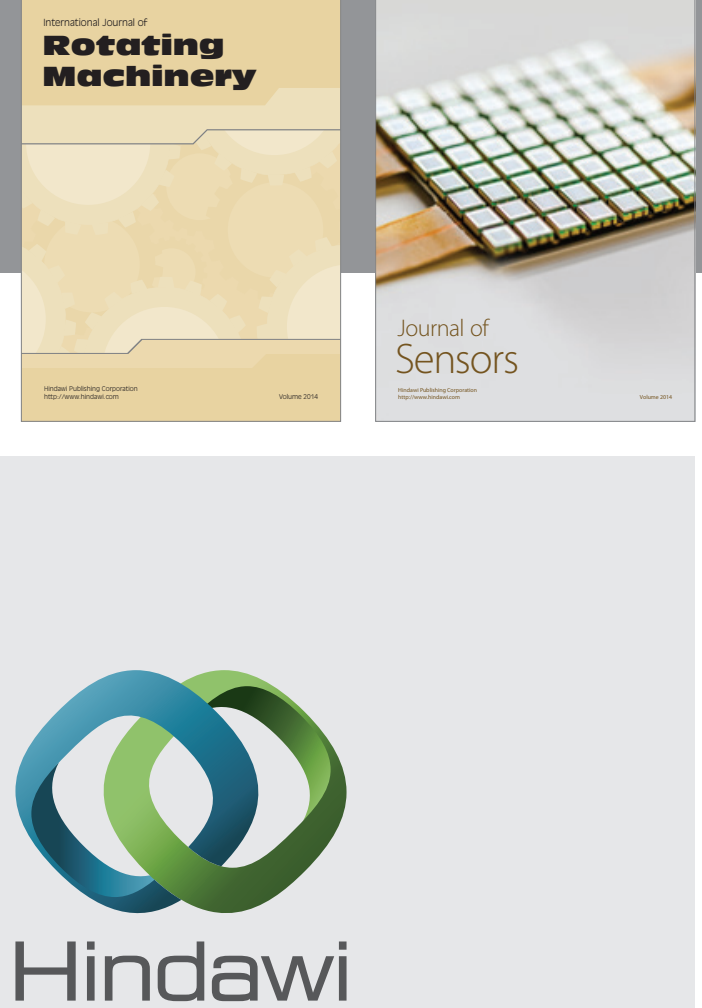

Submit your manuscripts at http://www.hindawi.com
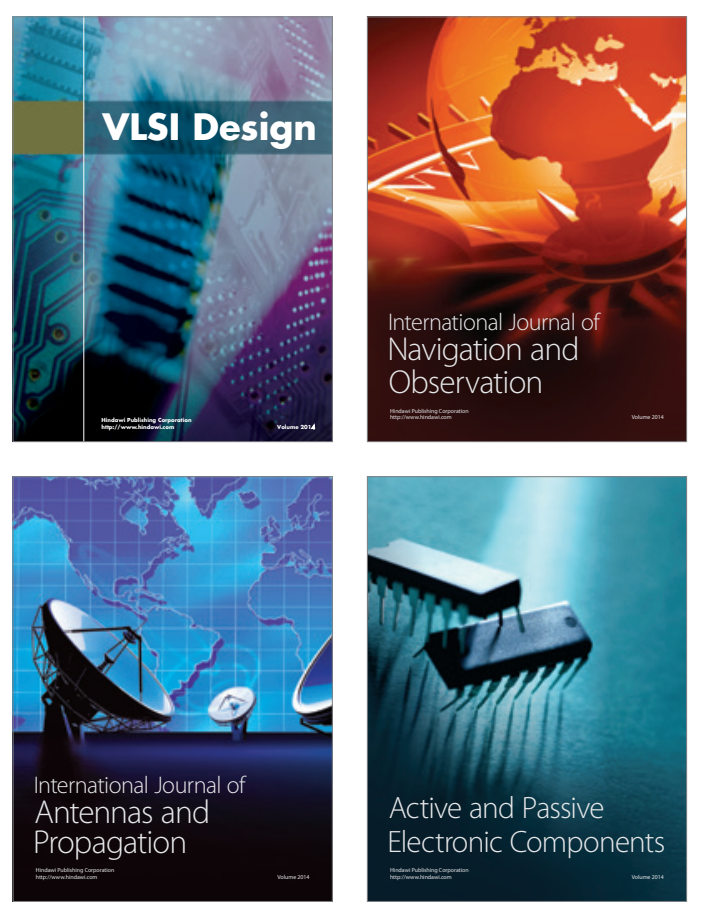
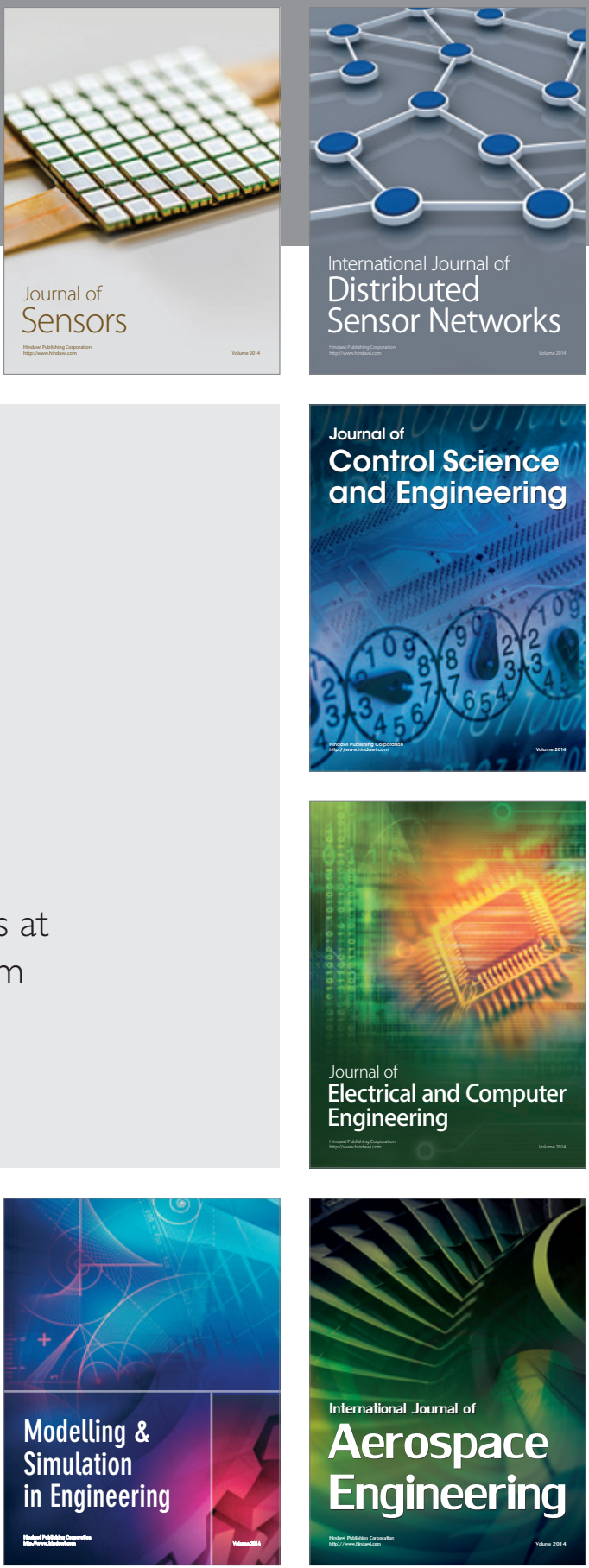

Journal of

Control Science

and Engineering
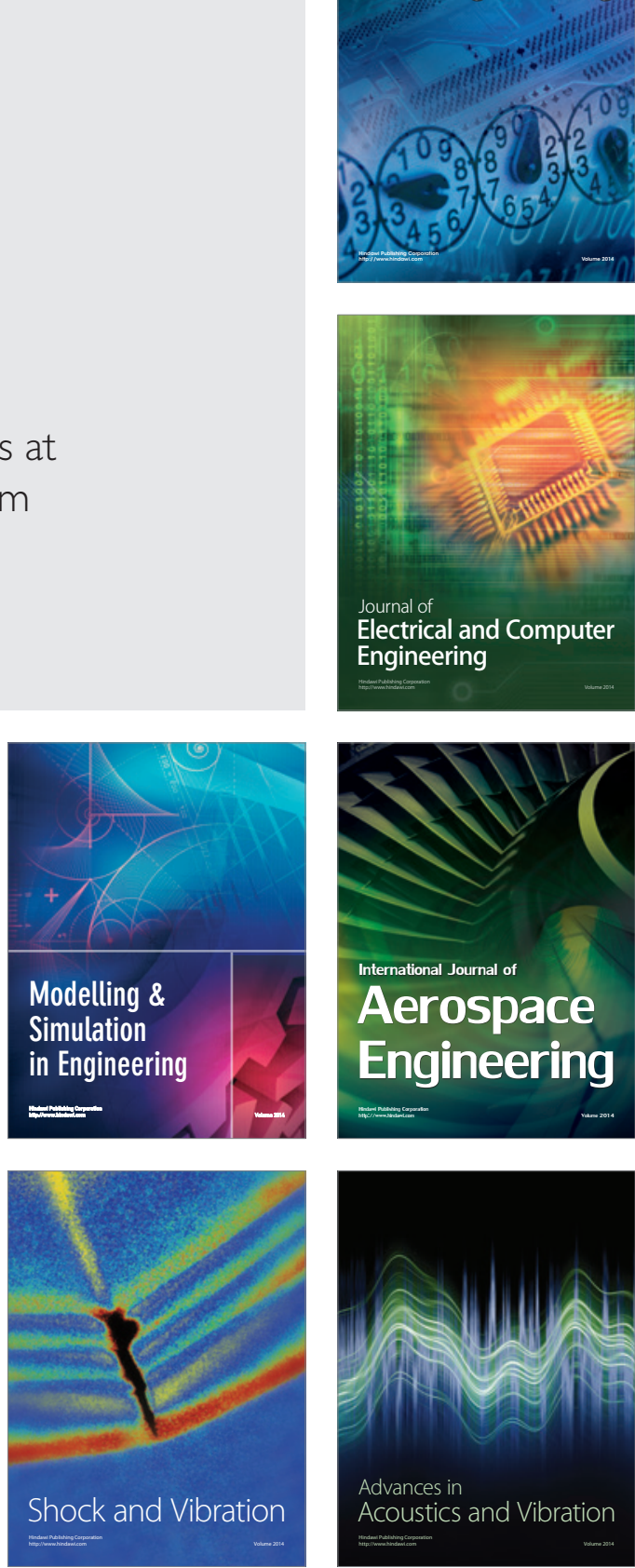